\title{
A Rapid Method for Magnetic Tracker Calibration Using a Magneto-optic Hybrid Tracker
}

\author{
Kazuhisa Nakada ${ }^{1}$, Masahiko Nakamoto ${ }^{1}$, Yoshinobu Sato ${ }^{1}$, \\ Kozo Konishi ${ }^{2}$, Makoto Hashizume ${ }^{2}$, and Shinichi Tamura ${ }^{1}$ \\ 1 Division of Interdisciplinary Image Analysis, Osaka University Graduate School of \\ Medicine, D11, 2-2 Yamadaoka, Suita City, Osaka 565-0871, JAPAN \\ 2 Graduate School of Medical Sciences, Kyushu University
}

\begin{abstract}
Electromagnetic trackers are greatly affected by magnetic field distortion resulting from metal objects and electronic equipment in close proximity. In this paper, we propose a rapid method for magnetic tracker calibration using a magneto-optic hybrid tracker. Although the calibration data can be acquired by freehand using the hybrid tracker, two problems arise as a result of freehand acquisition. One is the error caused by the time delay between the measurements of optical and magnetic trackers. The other is the inconsistency of calibration accuracy resulting from variations on uniformity and density of the freehand acquisition of calibration data. To overcome the problem of error, we developed a temporal calibration procedure to estimate the time delay and apply it accordingly. For the problem of inconsistent calibration accuracy, we formulated a distortion model selection method using a cross-validation technique with five distortion models obtained by 0 -th to 4 -th degree polynomial fitting to the calibration data. We experimentally evaluated the method in a setting affected by an actual operating table. By combining the temporal calibration and model selection methods, the error caused by the magnetic distortion was reduced from around $40 \mathrm{~mm}$ to less than $2 \mathrm{~mm}$, and 30 seconds were needed to obtain the calibration data for a $200 \times 200 \times 20 \mathrm{~mm}^{3}$ volume.
\end{abstract}

\section{Introduction}

Six-degree-of-freedom (6D) magnetic trackers measure the position and the orientation of a magnetic receiver relative to a magnetic field generator. The magnetic tracking systems have been the most widely used technology in virtual reality research and applications. However, their accuracy is greatly affected by magnetic field distortion resulting from the presence of electrically or magnetically active elements near the field generator or the receiver. In the past decade, researchers proposed a number of techniques to calibrate magnetic tracker accuracy by compensating magnetic field distortion [12].

Our task of magnetic distortion correction is to calibrate a magnetic tracker in an operation theatre setting where various metal objects and electronic equip- 
ment exist. In such an environment, when the operating table is electrically powered, the error due to the distortion can be as large as several centimeters. In addition, we are currently developing a system for laparoscopically augmented reality visualization with $3 \mathrm{D}$ ultrasound images. In order to obtain $6 \mathrm{D}$ pose parameters of an ultrasound (US) probe for laparoscopic 3D US systems [3], magnetic tracking is effective because the US probe can flexibly move inside the abdominal cavity while optical tracking is difficult due to occlusion of lines of sight. To attain high degree of accuracy, magnetic distortion correction is necessary.

The distortion correction is performed by approximating a distortion model through fitting the calibration data set of both distorted and undistorted measurements on the same point to certain analytical function, typically a polynomial. A review on published distortion correction techniques indicates that undistorted data are typically corrected using a mechanical or an optical tracker 45]. Previous studies [12] focus on obtaining accurate distortion models for a large space such as a $1 \times 1 \times 1 \mathrm{~m}^{3}$ volume using calibration data acquired based on a carefully planned acquisition protocol. Thus, they are not suitable for intraoperation applications which require a reduced amount of time and effort.

In this paper, we propose a rapid method for magnetic distortion correction which minimizes the time and effort for calibration data acquisition so as to be intraoperatingly applicable. In the proposed method, a magneto-optic hybrid tracker (hereafter, "hybrid tracker") [6 7] is attached to a data collection apparatus. The calibration data are collected by moving the apparatus via freehand inside the space where a distortion model needs to be approximated. Although the freehand data collection is particularly suitable for our purpose, two problems arise. One is the error caused by the time delay between the measurements of optical and magnetic trackers. The other is the inconsistency of calibration accuracy resulting from variations on uniformity and density of the freehand acquisition of calibration data. In the published studies 12 , the error problem is not addressed even when the calibration data are acquired by freehand. This problem could become serious when the apparatus is moved speedily for rapid collection. To address the issue, we develop a temporal calibration procedure to estimate the time delay using time-stamp and apply it accordingly. The inconsistent calibration accuracy is not considered either in the previous studies because the calibration data are assumed to be collected densely on regular grid. To overcome this problem, we formulate a distortion model selection method using a cross-validation technique with five distortion models obtained by 0 -th to 4-th degree polynomial fitting to the calibration data.

\section{Methods}

\subsection{Magneto-optic Hybrid Tracker}

A $6 \mathrm{D}$ optical tracker is modeled as a system measuring transformation $T_{\text {ot } \rightarrow o m}$ from optical tracker coordinate system $\Sigma_{\text {ot }}$, whose center is the optical camera, to optical marker coordinate system $\Sigma_{o m}$. A $6 \mathrm{D}$ magnetic tracker is modeled as 


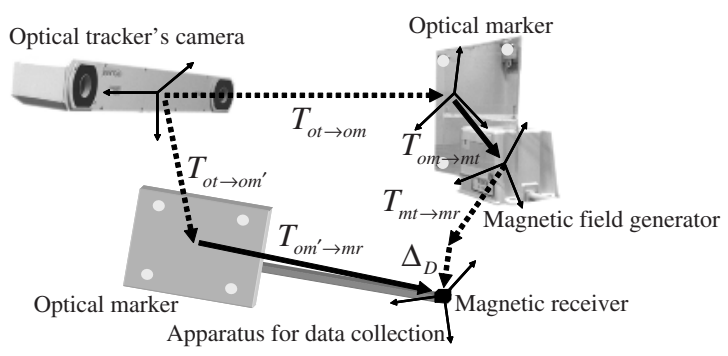

Fig. 1. Experimental setup used for calibration data acquisition by freehand. Dashed arrows denote dynamically changeable transformation, and solid arrows denote fixed transformation obtained beforehand by calibration.

a system measuring transformation $T_{m t \rightarrow m r}$ from magnetic tracker coordinate system $\Sigma_{m t}$, whose center is the magnetic field generator, to magnetic receiver coordinate system $\Sigma_{m r}$.

In order to register the magnetic and optical tracker coordinate systems, an optical marker $\Sigma_{o m}$ is attached to the field generator $\Sigma_{m t}$. Fixed transformation $T_{o m \rightarrow m t}$ from $\Sigma_{o m}$ to $\Sigma_{m t}$ is obtained beforehand by a calibration process in an environment without magnetic distortion [7. Using $T_{o m \rightarrow m t}$, transformation $T_{o t \rightarrow m r}$ from the optical camera $\Sigma_{o t}$ to magnetic receiver $\Sigma_{m r}$ is written as

$$
T_{o t \rightarrow m r}=T_{o t \rightarrow o m} T_{o m \rightarrow m t} T_{m t \rightarrow m r}
$$

indicating that a magnetic receiver $\Sigma_{m r}$ is localized in the optical tracker coordinate system $\Sigma_{o t}$. This transformation is shown in the upper part of Fig. 1]

\subsection{Calibration Data Acquisition by Freehand}

The calibration data can be acquired by measuring the identical spatial point using both a magnetic tracker and an optical tracker under the registration of magnetic and optical tracker coordinate systems. Both an optical marker $\Sigma_{\text {om }}$ and a magnetic receiver $\Sigma_{m r}$ are attached to the apparatus for calibration data collection. Fixed transform $T_{o m^{\prime} \rightarrow m r}$ from $\Sigma_{o m^{\prime}}$ to $\Sigma_{m r}$ is obtained beforehand by a calibration process in an environment without magnetic distortion. Using $T_{o m^{\prime} \rightarrow m r}$, transformation $T_{o t \rightarrow m r}^{\prime}$ from the optical camera $\Sigma_{o t}$ to magnetic receiver $\Sigma_{m r}$ is written as

$$
T_{o t \rightarrow m r}^{\prime}=T_{o t \rightarrow o m^{\prime}} T_{o m^{\prime} \rightarrow m r} .
$$

This transformation is shown in the lower part of Fig. 1

As shown in Fig. [1] there are two pathways to localize the identical magnetic receiver $\Sigma_{m r}$ in the optical tracker coordinate system $\Sigma_{o t}, T_{o t \rightarrow m r}$ in Eq. (11) and $T_{o t \rightarrow m r}^{\prime}$ in Eq. (2). It should be noted that $T_{o t \rightarrow m r}$ in Eq. (1) measured via a hybrid tracker is affected by magnetic distortion, while $T_{o t \rightarrow m r}^{\prime}$ in Eq. (2) measured via an optical tracker is not affected. Thus, the calibration data $\Delta_{D}$ 


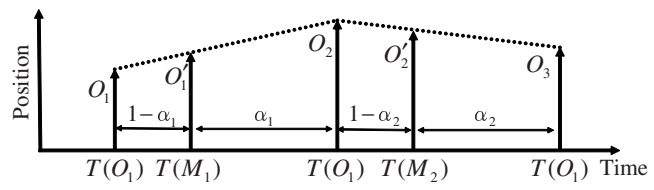

Fig. 2. Temporal calibration via linear interpolation.

representing the position and orientation error resulting from magnetic distortion is described as

$$
\Delta_{D}=T_{o t \rightarrow m r}^{-1} T_{o t \rightarrow m r}^{\prime}
$$

Freehand acquisition of the calibration data is obtained by moving the apparatus for data collection within the space where distortion correction is needed.

\subsection{Temporal Calibration for Freehand Acquisition}

In freehand acquisition, there is a time delay between the two measurements via a hybrid tracker and an optical tracker, i.e. $T_{o t \rightarrow m r}$ and $T_{o t \rightarrow m r}^{\prime}$, because of the moving apparatus during data collection. In the two pathways of data collection, $T_{o t \rightarrow o m^{\prime}}$ and $T_{m t \rightarrow m r}$ are affected by the movement of the apparatus. Let $\delta_{t}$ be the time delay between the optical tracker measurement $T_{o t \rightarrow o m^{\prime}}$ and the magnetic tracker measurement $T_{m t \rightarrow m r}$. By incorporating the time delay $\delta_{t}$, $T_{o t \rightarrow m r}^{\prime}$ is rewritten as

$$
T_{o t \rightarrow m r}^{\prime}=\left(T_{o t \rightarrow o m^{\prime}} \delta_{t} V\right) T_{o m^{\prime} \rightarrow m r}
$$

where $V$ denotes the velocity matrix of the apparatus for data collection. We utilize the above modified equation of $T_{o t \rightarrow m r}^{\prime}$ because the additional error due to the time delay may affect distortion correction when $V$ is large. To apply the modified equation, $\delta_{t}$ needs to be estimated beforehand by a temporal calibration process and $V$ can be estimated using time series of the measured positional data based on the assumption that the apparatus moves locally with a constant velocity.

We use the time-stamp of measurements for temporal calibration. Let $M_{i}$ and $T\left(M_{i}\right)$ be $i$-th measurement of a magnetic tracker and its time-stamp, respectively. Let $O_{i}$ and $T\left(O_{i}\right)$ be $i$-th measurement of an optical tracker and its time-stamp, respectively. If we assume that the time-stamp represents the exact time of measurement and the magnetic receiver moves locally with a constant velocity, the temporal calibration via linear interpolation (Fig. 2) is written as

$$
O_{i}^{\prime}=\left(1-\alpha_{i}\right) O_{i}+\alpha_{i} O_{i+1}, \quad \alpha_{i}=\frac{T\left(O_{i+1}\right)-T\left(M_{i}\right)}{T\left(O_{i+1}\right)-T\left(O_{i}\right)} .
$$

If the time delay between the time-stamp and the exact time is identical in both magnetic and optical measurements, the above calibration is still valid 
even though the time-stamp does not represent the exact time. In fact, under no practical circumstances is the delay identical. The time delay $\delta_{t}$ is defined as the difference of the time delay between magnetic and optical measurements.

The time delay $\delta_{t}$ is estimated by minimizing

$$
\sum_{i=1}^{n}\left(O_{i}^{\prime}-M_{i}\right)^{2}
$$

where

$$
O_{i}^{\prime}=\left(1-\alpha_{i}\right) O_{i}+\alpha_{i} O_{i+1}, \quad \alpha_{i}=\frac{\left(T\left(O_{i+1}\right)-\delta_{t}\right)-T\left(M_{i}\right)}{\left(T\left(O_{i+1}\right)-\delta_{t}\right)-\left(T\left(O_{i}\right)-\delta_{t}\right)} .
$$

The data collection for the above least squares is performed by moving the apparatus shown in Fig. [1 in an environment without magnetic distortion.

\subsection{Selection of Distortion Model by Cross-Validation}

Because the position and orientation of magnetic field distortion is independent [2], $\Delta_{D}$ can be decomposed into position vector $\mathbf{p}_{e}$ and orientation vector $\mathbf{q}_{e}$ represented as the vector part of a quaternion [2]. Further, because the distortion is considered to depend only on the position of the magnetic receiver [2], the calibration data samples $\mathbf{p}_{e}^{i}$ and $\mathbf{q}_{e}^{i}$ are independently fitted to $r$-the degree polynomial [5], where $r=0,1,2,3,4$. Our purpose is to select the best model among 0-th, 1-st, 2-nd, 3-rd, and 4-th degree polynomials, that is most adapted for the variation of the distortion where the data are collected as well as for the conditions, such as uniformity and density, of the calibration data samples by freehand acquisition. We assume that calibration data samples are not necessarily dense and uniform because their collection is rapidly performed by freehand in an operating theatre setting. Thus, high degree polynomials often suffer from instability although 4-th degree polynomial is common for uniformly and densely sampled data for a large space [8].

We use a cross-validation technique for the model selection. Two data sets for calibration are separately acquired for the same space, When one is used for model estimation, the other is used for model validation, and vice versa. Let $V=\left\{\mathbf{v}_{i} \mid i=1 \ldots m\right\}$ be the first data set, and $V^{\prime}=\left\{\mathbf{v}_{i}^{\prime} \mid i=1 \ldots n\right\}$ be the second data set. Let $\left\{M_{j} \mid j=0 \ldots 4\right\}$ and $\left\{M_{j}^{\prime} \mid j=0 \ldots 4\right\}$ represent estimated $j$-th degree polynomial using the first and second data set, respectively. We use a "goodness-of-fit" measure for the model selection based on $\epsilon=\frac{1}{n} \sum_{i=1}^{n}\left|\mathbf{v}_{i}^{\prime}-\hat{\mathbf{v}}_{i}\right|$ and $\epsilon^{\prime}=\frac{1}{m} \sum_{i=1}^{m}\left|\mathbf{v}_{i}-\hat{\mathbf{v}}_{i}^{\prime}\right|$, where $\hat{\mathbf{v}}_{i}$, and $\hat{\mathbf{v}}_{i}^{\prime}$ denote the distortion vectors at the same position as $\mathbf{v}_{i}$ and $\mathbf{v}_{i}^{\prime}$, which are obtained from each distortion model $M_{j}$ and $M_{j}^{\prime}$, respectively. The cross-validation procedure using $\epsilon$ and $\epsilon^{\prime}$ is as follows:

1. Estimate $\left\{M_{j} \mid j=0 \ldots 4\right\}$ using $V$.

2. Validate $\left\{M_{j} \mid j=0 \ldots 4\right\}$ using $V^{\prime}$, and obtain $\{\epsilon\}$.

3. Estimate $\left\{M_{j}^{\prime} \mid j=0 \ldots 4\right\}$ using $V^{\prime}$.

4. Validate $\left\{M_{j}^{\prime} \mid j=0 \ldots 4\right\}$ using $V$, and obtain $\left\{\epsilon^{\prime}\right\}$. 


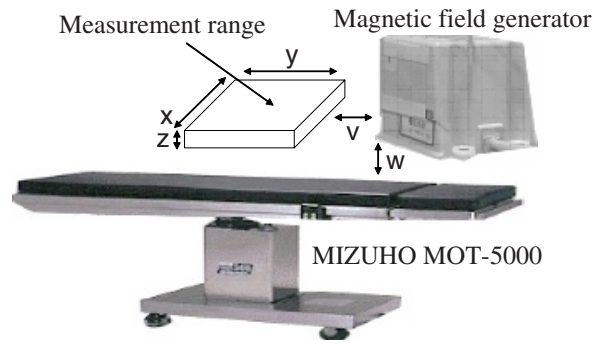

Fig. 3. Experimental setting of magnetic distortion correction.

5. Select $j_{o p t^{\text {th }}}$ degree polynomial model, which minimizes $\epsilon+\epsilon^{\prime}$.

6. Estimate the distortion model by $j_{\text {opt }}$-th degree polynomial fitting using $V+V^{\prime}$.

\section{Experimental Results}

\subsection{Experimental Conditions and Evaluation Methods}

In the experiments MiniBIRD (Ascension Technology Ltd., Burlington, VT, USA) was employed as a magnetic tracker. Polaris (Northern Digital Inc., Waterloo, Ontario, Canada) was employed as an optical tracker. Figure 3 shows the configuration used in the experiments for the evaluation of the model selection procedure, where MOT-5000 (MIZUHO Ltd. Tokyo, Japan) was used for the operating table which is electrically powered in order to reproduce an operating theatre setting. The measurement range and its position relative to the operating table were chosen based on the actual conditions in a laparoscopic liver surgery. The position of the field generator also resembled the real situation. We set the following values for the lengths in Fig. [3: $x=200 \mathrm{~mm}, y=200 \mathrm{~mm}, z=20$ $\mathrm{mm}, v=200 \mathrm{~mm}, w=200 \mathrm{~mm}$.

We defined "estimated accuracy" obtained by cross-validation as

$$
\delta_{\text {estimated }}=\frac{\epsilon+\epsilon^{\prime}}{2}
$$

where $\epsilon$ and $\epsilon^{\prime}$ are obtained in the cross-validation procedure described in Section 2.4. We also defined "actual accuracy" as

$$
\delta_{\text {actual }}=\frac{1}{N} \sum_{i=1}^{N}\left|\overline{\mathbf{v}}_{i}-\hat{\mathbf{v}}_{i}\right|,
$$

where $N$ is the number of data. $\overline{\mathbf{v}}_{i}$ is a distortion data set for the evaluation of the "estimated accuracy", which was a separate data set carefully sampled 


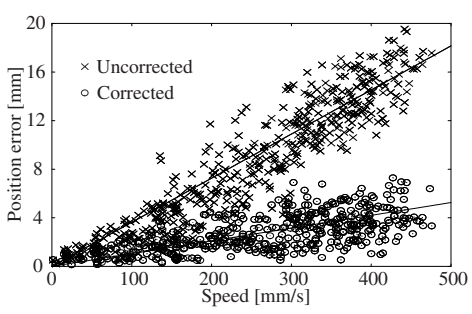

(a)

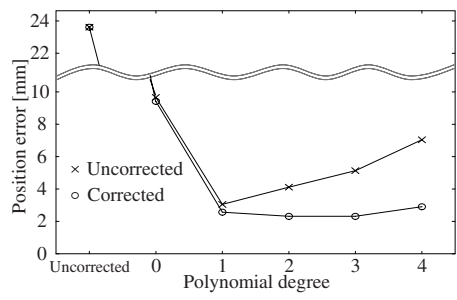

(b)

Fig. 4. Results of temporal calibration. Corrected: With temporal calibration. Uncorrected: Without temporal calibration. (a) Effects of speed on the accuracy of temporal calibration. (b) Effects of polynomial degree on the accuracy of final distortion correction results when speed is $200 \mathrm{~mm} / \mathrm{sec}$.

on a dense grid in the measurement range. $\hat{\mathbf{v}}_{i}$ is the distortion vector at the same position as $\overline{\mathbf{v}}_{i}$, which is obtained from the estimated distortion model. The "estimated accuracy" was a "goodness-of-fit" measure computed in the proposed method. The "actual accuracy" was defined in order to objectively evaluate the reliability of "estimated accuracy".

\subsection{Temporal Calibration}

The time delay $\delta_{t}$ was estimated in an environment without magnetic field distortion. The data for estimating $\delta_{t}$ was collected by freehand using the apparatus shown in Fig. 1 Using 945 data points collected with the speed between 10 $\mathrm{mm} / \mathrm{sec}$ and $500 \mathrm{~mm} / \mathrm{sec}, \delta_{t}=28$ (msec) was estimated by least squares of Eq. (6). We repeated the estimation using different data sets several times, and the standard deviation of the estimated $\delta_{t}$ was 3.9 (msec). $\delta_{t}=28$ (msec) was then used in the subsequent evaluations.

Fig. 4(a) shows the positional errors with and without the temporal calibration. We collected the reference data sets to compute the positional error in the same manner as we did the data sets for calibration. The error was significantly reduced, especially at high speed. For the speed of $300 \mathrm{~mm} / \mathrm{s}$, the error was reduced from $11.8 \mathrm{~mm}$ to $4.4 \mathrm{~mm}$. Even for the moderately slow speed of 150 $\mathrm{mm} / \mathrm{s}$, the error was reduced from $5.9 \mathrm{~mm}$ to $2.2 \mathrm{~mm}$.

Fig. 4(b) shows the "actual error" $\delta_{\text {actual }}$ for the distortion models of different polynomial degrees when the speed is $200 \mathrm{~m} / \mathrm{sec}$. The distortion models were estimated in an environment with magnetic distortion. For the second degree polynomial model, the error was reduced from $4.11 \mathrm{~mm}$ to $2.31 \mathrm{~mm}$. For fourth degree, the error was reduced from $7.04 \mathrm{~mm}$ to $2.90 \mathrm{~mm}$. Temporal calibration was effective especially for higher degree polynomial models. 


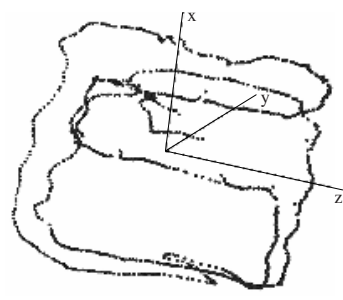

(a)

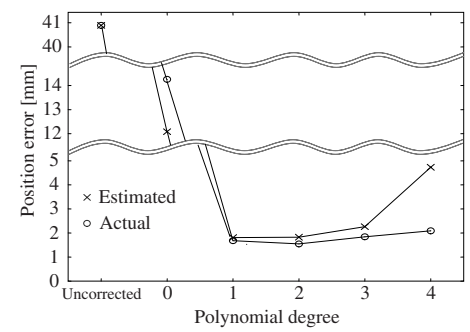

(b)

Fig. 5. Results of model selection. (a) Trajectory of collected calibration data by freehand. (b) Comparison between "actual" and "estimated" accuracies.

\subsection{Model Selection}

The distortion models were estimated in the experimental conditions shown in Fig. 3. The distortion model with the optimal polynomial degree was selected using the "estimated accuracy" $\delta_{\text {estimated }}$ via the cross-validation procedure. The average speed of the apparatus for data collection was $40 \mathrm{~mm} / \mathrm{sec}$. The number of the calibration data was 567 and 573 for first and second data sets, respectively. Fig. 罒(a) shows the trajectory of calibration data collected by freehand. It took about 30 seconds to obtain the calibration data. Fig. 5 (b) shows the comparison between the "actual" and "estimated" accuracies for different polynomial degrees. The "actual" accuracy was improved from more than $40 \mathrm{~mm}$ without any correction to less than $2 \mathrm{~mm}$ with first or second degree polynomial approximation. Although the best model was the first degree based on the "estimated" accuracy, the "estimated" accuracy of the second degree had almost the same value. The "actual" accuracy was the best at the second degree.

\section{Discussion and Conclusions}

We have proposed a magnetic distortion correction method for magnetic trackers, consisting of "temporal calibration" for freehand use of a magneto-optic hybrid tracker, which is employed for calibration data collection, and "model selection" of the best polynomial degree for distortion approximation based on a cross validation technique. The feature of the method is the robustness for fast and rough calibration data collection, and therefore it can be used in an operating theatre environment. In the experimental evaluations, temporal calibration is shown to be highly effective not only for the rapid data collection but also for higher degree polynomial approximation. In model selection, the proposed cross-validation procedure exhibits a tendency to select a slightly lower polynomial degree than that minimizing the actual accuracy. Such a tendency is also observed in additional experiments (data not shown). This feature would be favorable since preferring lower degree can be viewed as carrying out safer selection when the accuracies differ only slightly. 
Based on our experience, magnetic distortion is especially large around the central area of an operating table, which is situated just above an electric motor. In a laparoscopic surgery, the liver is positioned in close proximity to this central area, and thus, distortion is large. In our experimental evaluation using the actual operating table, the positional error caused by magnetic distortion could be reduced from more than $40 \mathrm{~mm}$ to less than $2 \mathrm{~mm}$. Therefore, the proposed method would be particularly useful when magnetic tracker of flexible surgical tools inside the abdomen is employed for operation. Future work will explore the clinical application of the proposed method for magnetic tracking in the laparoscopic 3D ultrasound system. We are currently modifying the data collection apparatus shown in Fig. 1 in order to enable its insertion into a laparoscopic trocar (a pipe connecting the abdominal cavity and outside to insert surgical tools). The modified apparatus would consist of an optical marker and a magnetic receiver located outside and inside the abdominal cavity, respectively. We will then test the feasibility of our method in the laparoscopic procedure using this modified apparatus.

Acknowledgement. This work was partly supported by JSPS Research for the Future Program JSPS-RFTF99I00903.

\section{References}

1. Zachmann G, Virtual reality in assembly simulation - collision detection, simulation algorithms, and interaction techniques, PhD Dissertation, TU Darmstadt, 2000.

2. Ikits $\mathrm{M}$, et al., An improved calibration framework for electromagnetic tracking devices, IEEE Virtual Reality 2001, 2001.

3. Nakamoto M, et al., 3D ultrasound system using a magneto-optic hybrid tracker for augmented reality visualization in laparoscopic liver surgery, Lect Notes Comput Sc, vol. 2489 (Proc. MICCAI 2002, Part II, Tokyo, Japan), pp. 148-155, 2002.

4. Livingston MA, et al., Magnetic tracker calibration for improved augmented reality registration, Presence of Teleoperators and Virtual Environment, vol. 6, pp. 532$546,1997$.

5. Kindratenko VV et al., A survey of electromagnetic position tracker calibration teckniques, Virtual Reality: Research, Development, and Applications, vol. 5, pp. 169-182, 2001

6. Birkfellner W, et al., Calibration of tracking systems in a surgical environment, vol. 17, 737-741, 1998.

7. Nakamoto M, et al., Magneto-optic hybrid 3D sensor for surgical navigation, Lect Notes Comput Sc, vol. 1935 (Proc. MICCAI 2000, Pittsburgh, PA, USA), pp. 839-848, 2000.

8. Nixon M, et al., The effects of metals and interfering fields on electromagnetic trackers, Presence: Teleoperators and Virtual Environments, vol. 7, pp. 204-218, 1998. 